\title{
Mechanical and Thermal Stresses in a FGPM Hollow Cylinder due to Radially Symmetric Loads
}

\author{
M. Jabbari, ${ }^{1}$ M. Meshkini, ${ }^{1}$ and M. R. Eslami² \\ ${ }^{1}$ Islamic Azad University, South Tehran Branch, Tehran, Iran \\ ${ }^{2}$ Department of Mechanical Engineering, Academy of Sciences, Amirkabir University of Technology, Tehran, Iran
}

Correspondence should be addressed to M. Jabbari, projectsjabbari@gmail.com

Received 25 May 2011; Accepted 21 June 2011

Academic Editor: J. Seok

Copyright (C 2011 M. Jabbari et al. This is an open access article distributed under the Creative Commons Attribution License, which permits unrestricted use, distribution, and reproduction in any medium, provided the original work is properly cited.

\begin{abstract}
The general solution of steady-state on one-dimensional Axisymmetric mechanical and thermal stresses for a hollow thick made of cylinder Functionally Graded porous material is developed. Temperature, as functions of the radial direction with general thermal and mechanical boundary-conditions on the inside and outside surfaces. A standard method is used to solve a nonhomogenous system of partial differential Navier equations with nonconstant coefficients, using complex Fourier series, rather power functions method and solve the heat conduction. The material properties, except poisson's ratio, are assumed to depend on the variable $r$, and they are expressed as power functions of $r$.
\end{abstract}

\section{Introduction}

Poroelasticity is a theory that models the interaction of deformation and fluid flow in a fluid-saturated porous medium. The deformation of the medium influences the flow of the fluid and vice versa. The theory was proposed by Biot $[1,2]$ as a theoretical extension of soil consolidation models developed to calculate the settlement of structures placed on fluid-saturated porous soils. The historical development of the theory is sketched by De Boer [3]. The theory has been widely applied to geotechnical problems beyond soil consolidation, most notably problems in rock mechanics. Detournay and Cheng [4] survey both these methods with special attention to rock mechanics. These include familiar analytical methods (displacement potentials, method of singularities) and computational methods (finite element and boundary element). Sandhu and Wilson [5] are acknowledged for pioneering the application of finite element techniques to poroelasticity. Detournay and Cheng [6] presented fundamentals of poroelasticity.

Abousleiman and Ekbote [7] presented the analytical solutions for inclined hollow cylinder in a transversely isotropic material subjected to thermal and stress perturbations, and they systematically evaluated the effect of the anisotropy of the poromechanical material parameters as well as thermal material properties on stress and porous pressure distributions. Chen [8] presented and analyzed the problems of linear thermo elasticity in a transversely isotropic hollow cylinder of finite length by a direct power series approximation through the application of the LanczosChebyshev method. Bai [9] presented then derived an analytical method solving the responses of a saturated porous media subjected to cyclic thermal loading by the Laplace transform and the Gauss-Lengender method of Laplace transform inversion. Wang and Papamichos [10, 11] presented analytical solution for the temperature, pore pressure, and stresses around a cylindrical well bore and a spherical cavity subjected to a constant fluid flow rate by coupling the conductive heat transfer with the pore-fluid flow. Ghassemi and Tao [12] presented influence of coupled chemo-poro-thermoelastic processes on pore pressure and stress distributions around a wellbore in swelling shale. Wirth and sobey [13] presented an axisymmetric and fully 3-D poroelastic model forth evolution of hydrocephalus. Yang and Zhang [14] presented poroelastic wave equation including the Biot/squirt mechanism and the solid/fluid coupling anisotropy. Arora and Tomar [15] presented the elastic waves along a cylindrical borehole in a poroelastic 


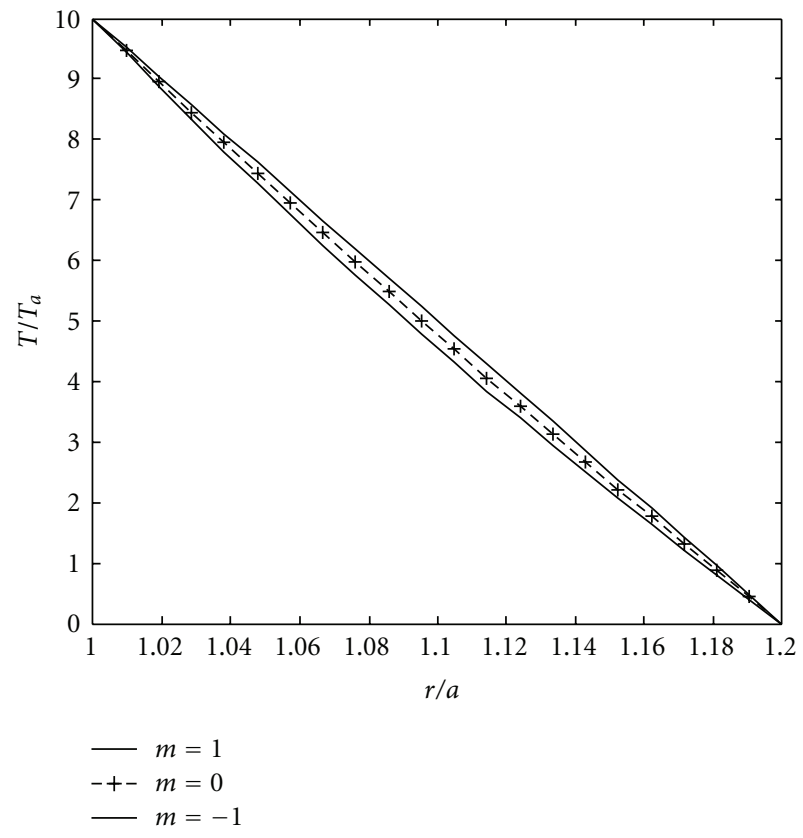

FIGURE 1: Temperature distribution in the cross-section of cylindrical.

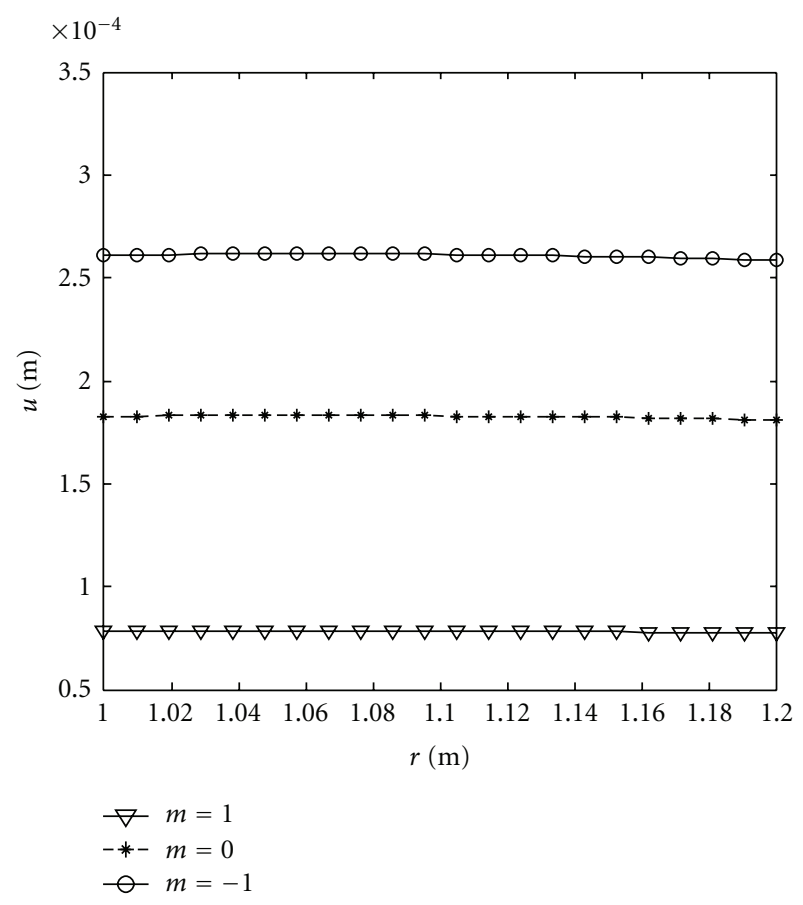

FIgURE 2: Radial displacement in the cross-section of cylindrical.

medium saturated by two immiscible fluids. Hamiel et al. [16] presented the coupled evolution of damage and porosity in poroelastic media theory and applications to the deformation of porous rocks. Ghassemi [17] presented stress and pore prepressure distribution around a pressurized, cooled crack in hollow permeability rock. Youssef [18] theory of generalized porothermoelasticity was presented. Jourine et al. [19] presented modeling poroelastic hollow cylinder experiments with realistic boundary conditions.

Functionally graded materials (FGMs) are heterogeneous materials in which the elastic and thermal properties change from one surface to the other, gradually and continuously. The material is constructed by smoothly changing materials. Since ceramic has good heat resistance and metal has high strength, ceramic-Metal FGM may work at super hightemperature or under high-temperature difference field. In effect, the governing equation for the temperature and stress distributions are coordinate dependent as the material properties are functions of position. Classical method of analysis is to combine the equilibrium equations with the stressstrain and strain-displacement relations to arrive at the governing equation in terms of the displacement components called the Navier equation. There are some analytical thermal and stress calculations for functionally graded material in the one-dimensional case for thick cylinders and spheres $[20,21]$. The authors have considered the nonhomogeneous material properties as liner function of $r$. Jabbari et al. [22] studied a general solution for mechanical and thermal stresses in a functionally graded hollow cylinder due to nonaxisymmetric steady-state load. They applied separation of variables and complex Fourier series to solve the heat conduction and Navier equation. Poultangari et al. [23] presented Functionally graded hollow spheres under nonaxisymmetric thermomechanical loads. Shariyat et al. [24] presented nonlinear transient thermal stress and elastic wave propagation analyses of thick temperature-dependent FGM cylinders, using a second-order point-collocation method. Lü et al. [25] presented elastic mechanical behavior of nanoscaled FGM films incorporating surface energies. Afsar and Sekine [26] presented inverse problems of material distributions for prescribed apparent fracture toughness in FGM coatings around a circular hole in infinite elastic media. Zhang and Zhou [27] presented a theoretical analysis of FGM thin plates based on physical neutral surface. Fazelzadeh and Hosseini [28] presented aerothermoelastic behavior of supersonic rotating thin-walled beams made of functionally graded materials. Ootao and Tanigawa [29] presented the transient thermoelastic problem of functionally graded thick strip due to nonuniform heat supply. They obtained the exact solution for the two-dimensional temperature change in a transient state, and thermal stress of a simple supported strip under the state of plane strain. Jabbari et al. [30] presented and studied the mechanical and thermal stresses in functionally graded hollow cylinder due to radially symmetric loads. They assumed the temperature distribution to be a function of radial direction. They applied a method to solve the heat conduction and Navier equations. Farid et al. [31] presented three-dimensional temperature dependentfree vibration analysis of functionally graded material curved panels resting on two-parameter elastic foundation using a hybrid semianalytic, differential quadrature method. Bagri and Eslami [32] presented Generalized coupled thermoelasticity of functionally graded annular disk considering the Lord-Shulman theory. Shariat and Eslami [33] presented buckling of thick functionally graded plates under 
mechanical and thermal loads. Jabbari et al. [34] studied an axisymmetric mechanical and thermal stresses in thick short length functionally graded material cylinder. They applied the separation of variables and complex Fourier series to solve the heat conduction and Navier equation. Thieme et al. [35] presented titanium powder sintering for preparation of a porous FGM destined as a skeletal replacement implant.

In this work, a direct method of solution of the Navier equations presented which does not have limitation of the potential function method as to handle the general type of mechanical and thermal under one-dimensional steady-state temperature distribution with general type of thermal and mechanical boundary conditions is considered. The functionally graded porous material properties of the cylinder are assumed to be expressed by power functions in $r$. The Naviear equation terms of displacements are derived and solved analytically by the direct method, so any boundary conditions for stresses and displacements can be satisfied.

Consider a hollow circular cylinder of inner radius $a$, outer radius $b$ made of functionally graded porous material (FGPM) respectively. Axisymmetric cylindrical coordinates $(r)$ are considered along the radial direction. The cylinder's material graded through the $r$ direction, thus the material properties are porous and functions of $r$. The first law of thermodynamics for energy equation in the steady-state condition for the FGPM on dimensional cylinder is:

$$
\begin{aligned}
& \frac{1}{r} \frac{\partial}{\partial r}\left[r k(r)\left(\frac{\partial T}{\partial r}\right)\right]=0 \longrightarrow \frac{\partial^{2} T}{\partial r^{2}}+\left(\frac{k^{\prime}(r)}{k(r)}+\frac{1}{r}\right) \frac{\partial T}{\partial r}=0, \\
& a \leq r \leq b \text {, }
\end{aligned}
$$

where $T(r)$ is temperature distribution, $k(r)$ is the thermal conduction coefficient, and symbol $(/)$ denotes derivative with respect to $r$.

\section{Heat Conduction Problem}

The thermal boundary is assumed as

$$
\begin{aligned}
& S_{11} T(a)+S_{12} T_{, r}(a)=f_{1}, \\
& S_{21} T(b)+S_{22} T_{, r}(b)=f_{2},
\end{aligned}
$$

where (,) denotes partial derivative, and $S_{i j}$ are the constant thermal parameters related to conduction and convection coefficients. We assume that nonhomogeneous thermal conduction coefficient $k(r)$ is power function of $r$ as $k(r)=k_{0} r^{m_{3}}$, where $k_{0}$ and $m_{3}$ material parameter. Using the definition for the material properties, the temperature equation becomes

$$
\frac{\partial^{2} T}{\partial r^{2}}+\left(m_{3}+1\right) \frac{1}{r} \frac{\partial T}{\partial r}=0
$$

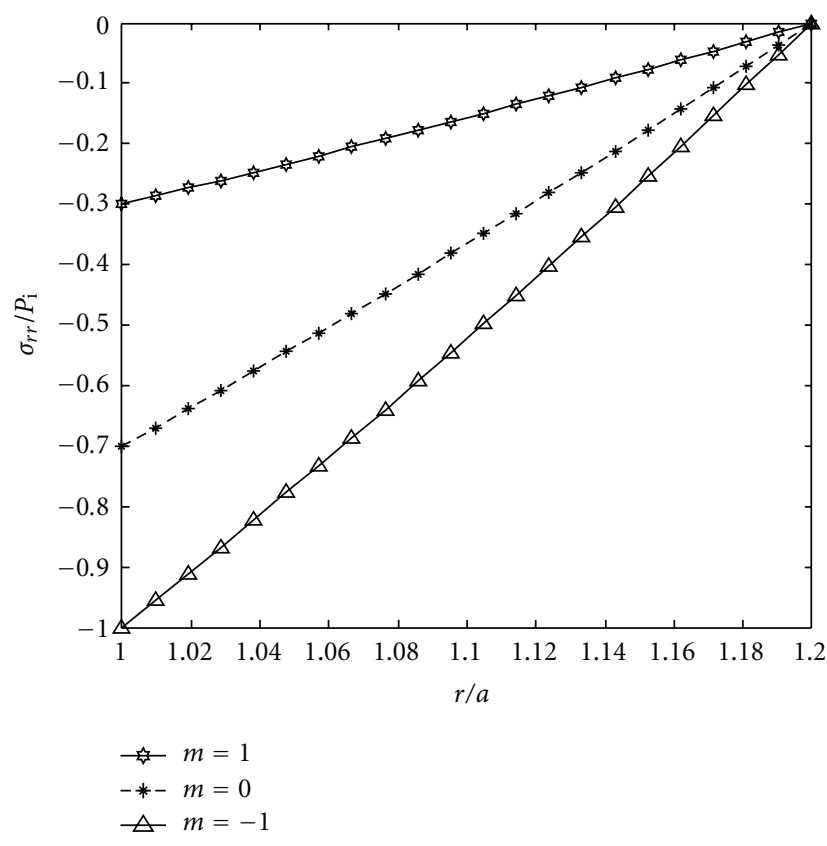

FIgURE 3: Radial distribution of radial stress.

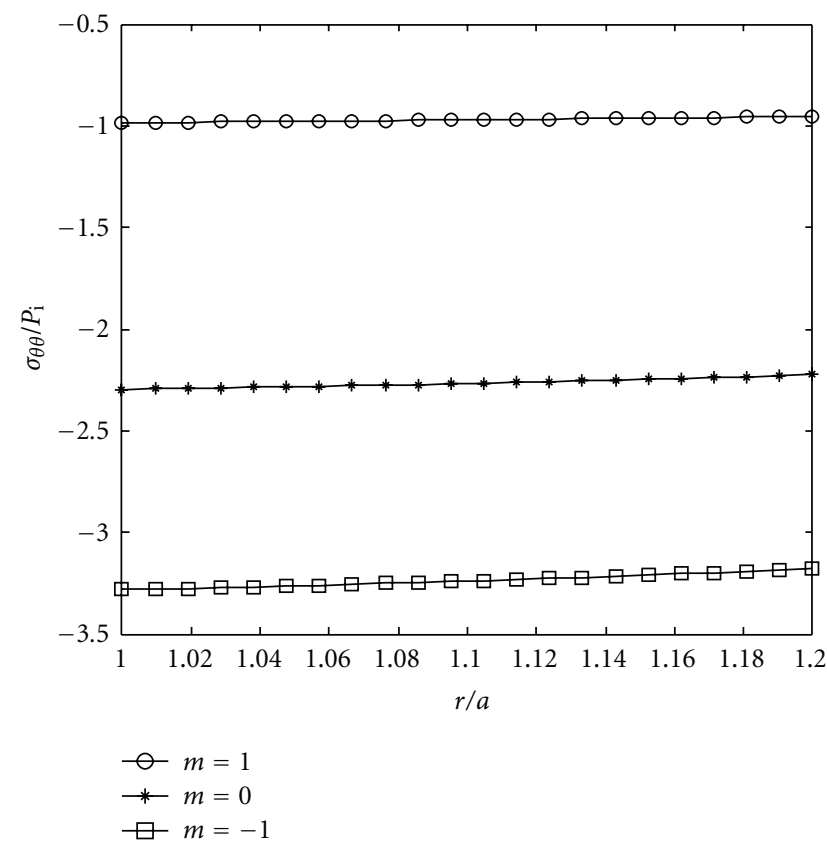

FIgURE 4: Radial distribution of hoop stress.

Integrating (4) twice yields

$\beta^{2}+\left(m_{3}+1\right) \beta=0 \longrightarrow\left\{\begin{array}{l}\beta_{1}=-\left(m_{3}+1\right) \\ \beta_{2}=0\end{array} \longrightarrow \beta=-\left(m_{3}+1\right)\right.$

$$
T(r)=\frac{a}{\beta+1} r^{\beta+1}+b \longrightarrow T(r)=\frac{-a}{m_{3}} r^{-m_{3}}+b
$$




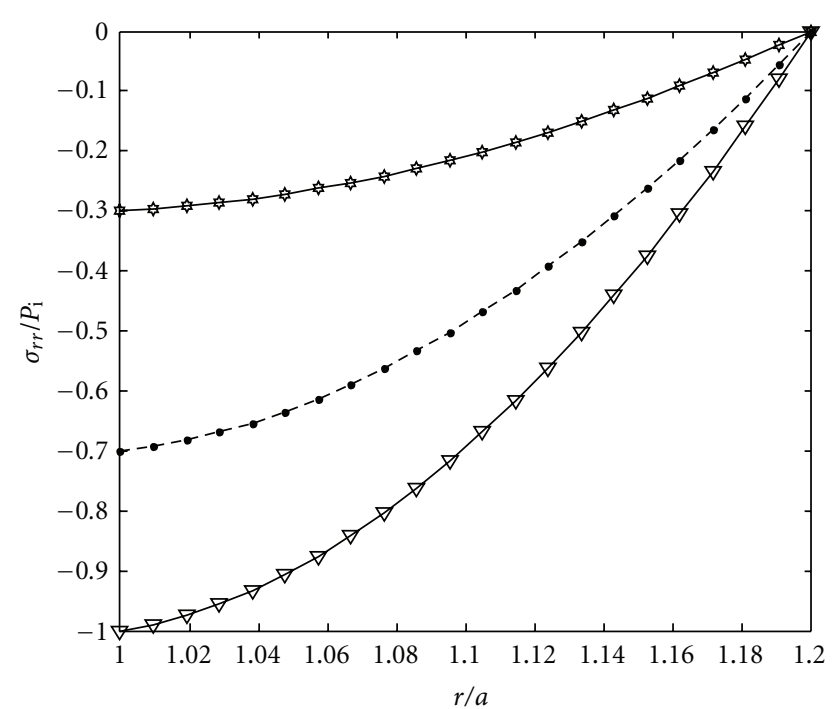

$B-B=0.85$

$-\bullet B=0.65$

$\rightarrow B=0.5$

FIGURE 5: Radial thermal stress in the cross-section of cylindrical based on the pore volume fraction $(B)$ changing.

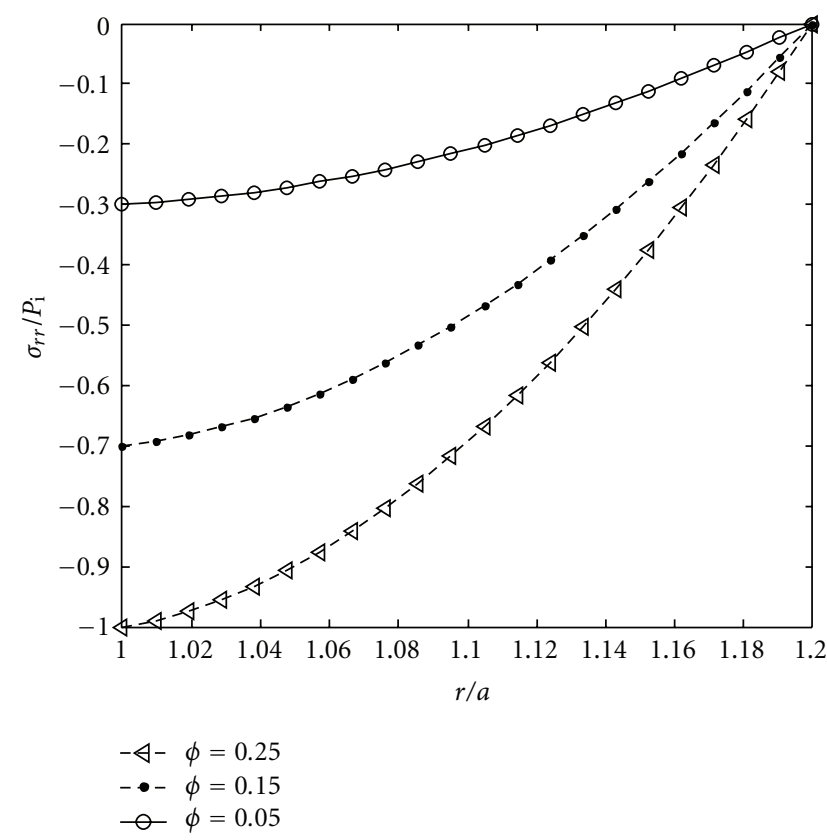

FIGURE 6: Radial thermal stress in the cross-section of cylindrical based on the pore volume fraction $(\phi)$ changing.

Using the boundary conditions (2) to determine the constants $a$ and $b$ yields

$$
A_{1}=\frac{e_{4} f_{1}-e_{2} f_{2}}{e_{1} e_{4}-e_{2} e_{3}}, \quad A_{2}=\frac{e_{1} f_{2}-e_{3} f_{1}}{e_{1} e_{4}-e_{2} e_{3}}
$$

where constants $e_{1}$ to $e_{4}$ are given in Appendix A.

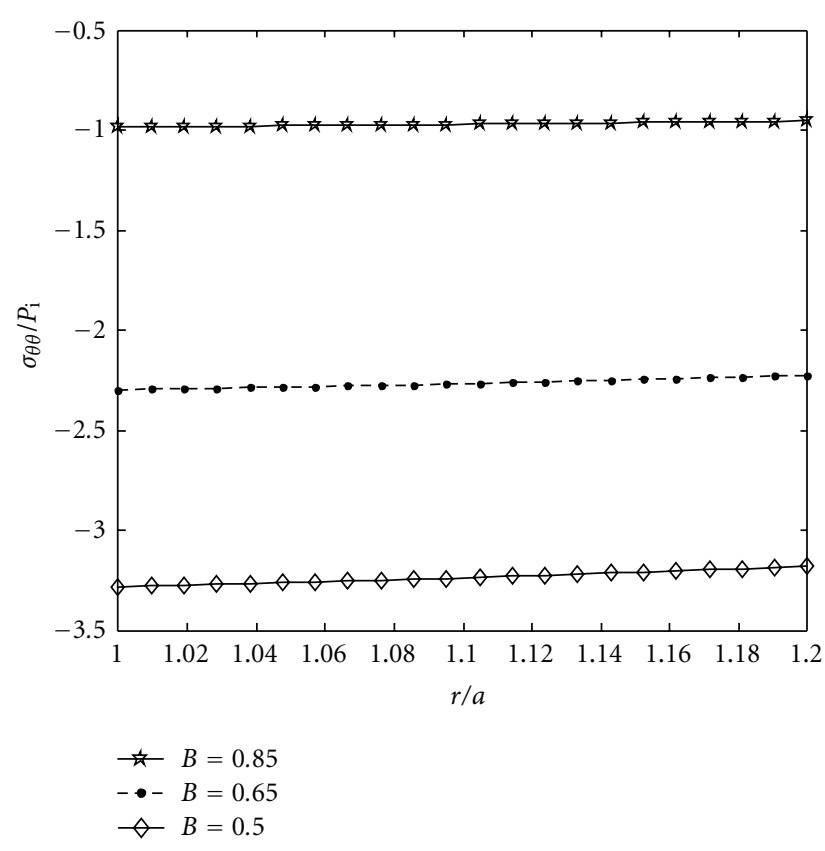

Figure 7: Hoop thermal stress in the cross section of cylindrical based on the compressibility coefficient $(B)$ changing.

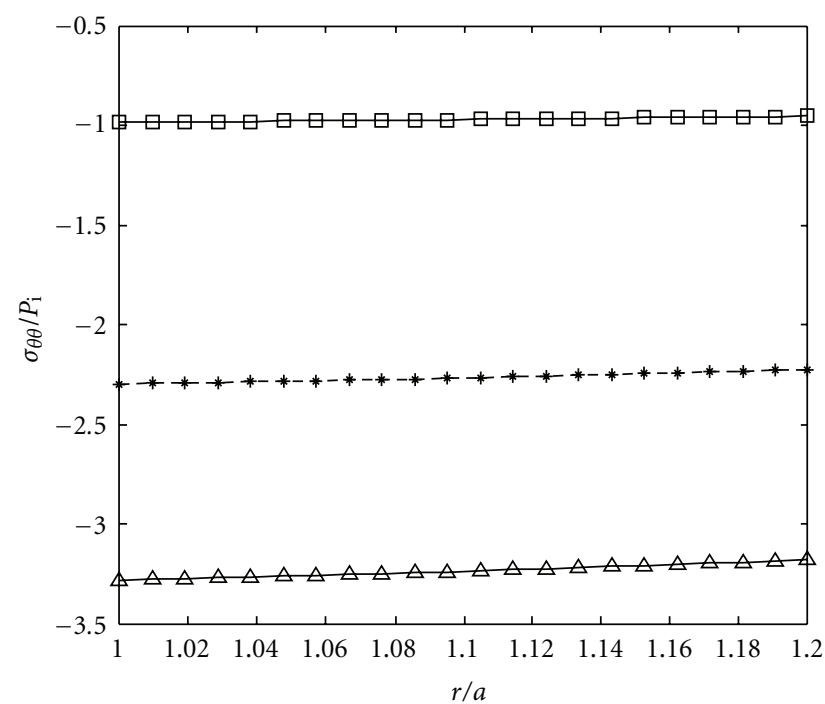

$\triangle \phi=0.25$

- *- $\phi=0.15$

$\square \phi=0.05$

Figure 8: Hoop thermal stress in the cross section of cylindrical based on the pore volume fraction $(\phi)$ changing.

\section{Stress Analysis}

Let $u$ displacement components in the radial direction. Then strain-displacement relations are

$$
\varepsilon_{r r}=\frac{\partial u}{\partial r}, \quad \varepsilon_{\theta \theta}=\frac{u}{r}
$$




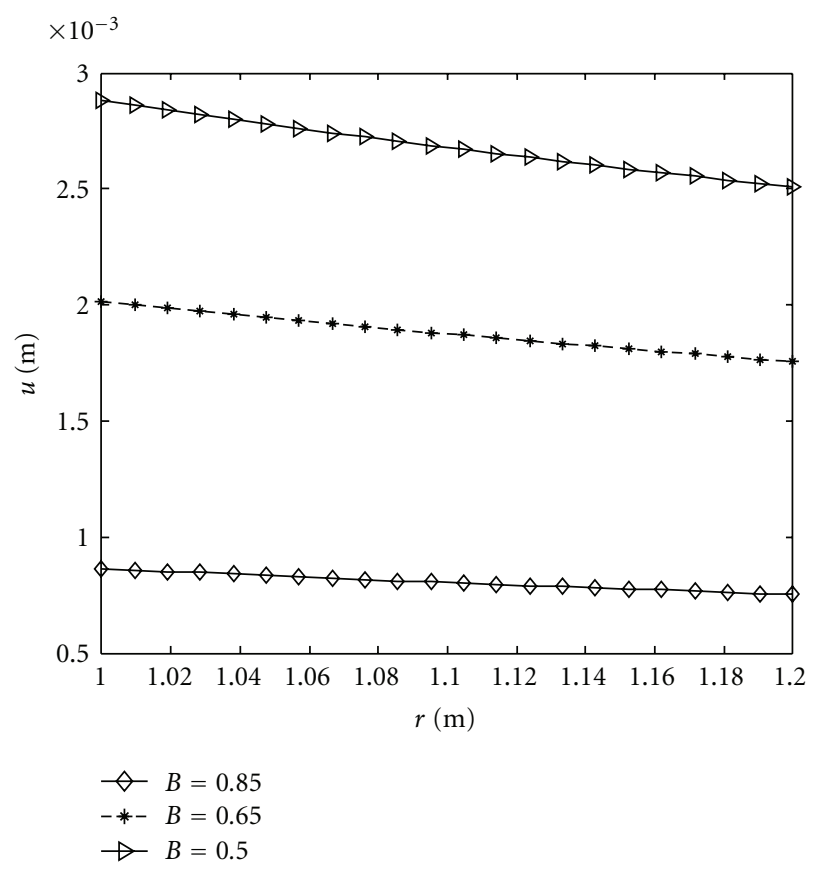

FIgURE 9: Radial displacement in the cross-section of cylindrical based on the compressibility coefficient $(B)$ changing.

and stress-strain relations of a functionally graded porous cylinder for nonaxisymmetric condition are

$$
\begin{gathered}
\sigma_{r r}=C_{11} \varepsilon_{r r}+C_{12} \varepsilon_{\theta \theta}-\gamma p \delta_{r r}-Z_{1} T(r), \\
\sigma_{\theta \theta}=C_{22} \varepsilon_{r r}+C_{12} \varepsilon_{\theta \theta}-\gamma p \delta_{\theta \theta}-Z_{2} T(r), \\
C_{11}+M \gamma^{2}=\stackrel{*}{C}_{11} \\
C_{22}+M \gamma^{2}=\stackrel{*}{C}_{22}, \\
C_{12}+M \gamma^{2}=\stackrel{*}{C}_{12}
\end{gathered}
$$

where $\sigma_{i j}, \in_{i j}(i, j=r, \theta), M, \gamma, \alpha, \lambda, \mu$, and $p$ are stress tensors, strain tensors, Biot's modulus, Biot's coefficient of effective stress, thermal expansion coefficient, lame's coefficient, and the pore pressure, respectively, $p$ related to the Biot's modulus, volumetric strain and the variation of fluid content.

We assume that pore-cylinder if undrained condition then $(\zeta=0)$ as:

$$
p=M\left(\zeta-\gamma\left(\varepsilon_{r r}+\varepsilon_{\theta \theta}\right)\right)=-M \gamma\left(\varepsilon_{r r}+\varepsilon_{\theta \theta}\right),
$$

where:

$$
M=\frac{2 \mu\left(\nu_{u}-\nu\right)}{\gamma^{2}(1-2 \nu)\left(1-2 v_{u}\right)} .
$$

Thus,

$$
\begin{aligned}
\sigma_{r r} & =\stackrel{*}{C}_{11} \varepsilon_{r r}+\stackrel{*}{C}_{12} \varepsilon_{\theta \theta}-Z_{1} T(r), \\
\sigma_{\theta \theta} & =\stackrel{*}{C}_{22} \varepsilon_{\theta \theta}+\stackrel{*}{C}_{21} \varepsilon_{r r}-Z_{2} T(r),
\end{aligned}
$$

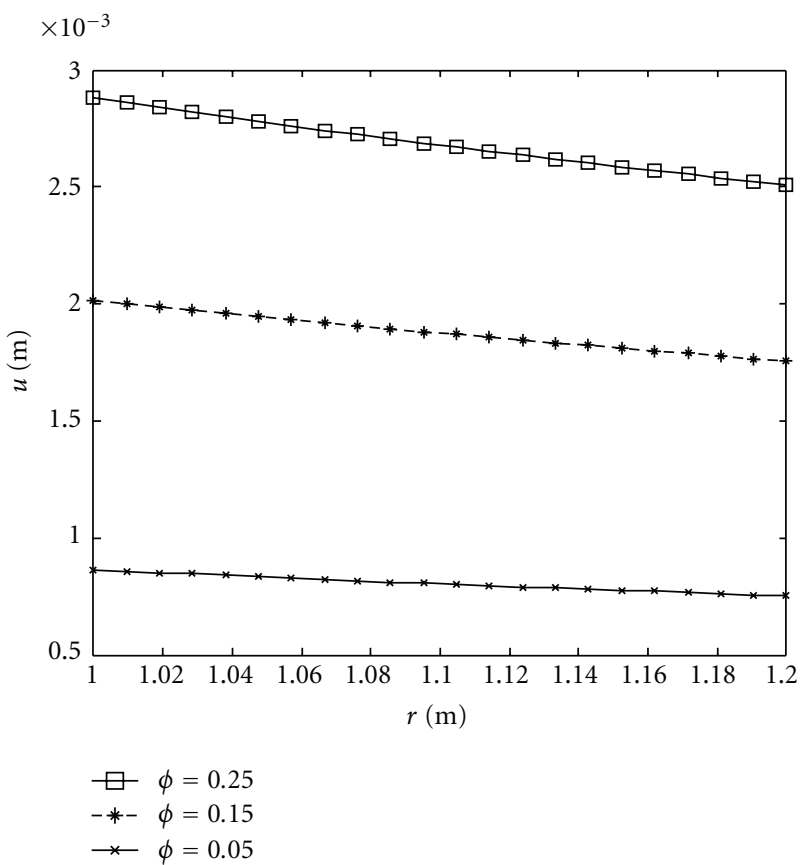

FIGURE 10: Radial displacement in the cross-section of cylindrical based on the compressibility coefficient $(\phi)$ changing.

The equilibrium equation in the radial direction, disregarding body force and the inertia terms, is

$$
\begin{gathered}
\frac{\partial \sigma_{r r}}{\partial r}+\frac{1}{r}\left(\sigma_{r r}-\sigma_{\theta \theta}\right)=0, \\
Z_{1}=\stackrel{*}{C}_{11} \alpha_{r}+2 \stackrel{*}{C}_{12} \alpha_{\theta}, \\
Z_{2}=2 \stackrel{*}{C}_{21} \alpha_{r}+\stackrel{*}{C}_{22} \alpha_{\theta} .
\end{gathered}
$$

To obtain the equilibrium equations in terms of the displacement components for the FGPM cylinder, the functional relationship and pore of the material properties must be known. Because the cylinder material is assumed to be graded along the $r$-direction, the modulus of elasticity and coefficient of thermal expansion are material constant

assumed to be described with the power laws as

$$
\alpha_{r}=\alpha_{01} r^{m_{1}}, \quad \alpha_{\theta}=\alpha_{02} r^{m_{2}}, \quad K=k_{0} r^{m_{3}}, \quad C_{i j}=\bar{C}_{i j} r^{m 4},
$$

where the coefficients are described as

$$
\alpha_{01}=\frac{\alpha_{01}^{\prime}}{a^{m_{1}}}, \quad \alpha_{02}=\frac{\alpha_{02}^{\prime}}{a^{m_{2}}}, \quad K=\frac{k_{0}^{\prime}}{a^{m_{3}}}, \quad \bar{C}_{i j}=\frac{\bar{C}_{i j}^{\prime}}{a^{m 4}},
$$

and $a$ is the inner radius. 
TABle 1

\begin{tabular}{lcccccccc}
\hline Parameters & $C_{11}$ & $C_{12}$ & $C_{22}$ & $\gamma$ & $E_{0}$ & $\nu$ & $\nu_{u}$ & $\alpha_{01}, \alpha_{02}$ \\
\hline Numerical & $139 \mathrm{GPa}$ & $78 \mathrm{GPa}$ & $139 \mathrm{GPa}$ & 0.47 & $200 \mathrm{GPa}$ & $0.2 \mathrm{GPa}$ & $0.3 \mathrm{GPa}$ & $0.000012\left(1 /{ }^{\circ} \mathrm{C}\right)$ \\
\hline
\end{tabular}

Using the relations (7) to (14), the Navier equations in terms of the displacement components are

$$
\begin{gathered}
\left(\frac{\partial^{2} u}{\partial r^{2}}+\left(m_{4}+1\right) \frac{1}{r}\left(\frac{\partial u}{\partial r}\right)+\left(\frac{\left(m_{4}+1\right) \bar{C}_{12}-\bar{C}_{22}}{\bar{C}_{11}}\right) \frac{1}{r^{2}} u\right) \times r^{m_{4}} \\
=\left(\left\{\left[\frac{\left(m_{1}+m_{4}-1\right) \bar{C}_{11}-2 \bar{C}_{12}}{\bar{C}_{11}}\right] \times r^{m_{1}-1} \alpha_{01}\right.\right. \\
\left.+\left[\frac{2\left(m_{2}+m_{4}+1\right) \bar{C}_{12}+\bar{C}_{22}}{\bar{C}_{11}}\right] \times r^{m_{2}-1} \alpha_{02}\right\} T(r, \theta) \\
\left.+\left[\frac{\bar{C}_{11} r^{m_{1}} \alpha_{01}+2 \bar{C}_{12} r^{m_{2}} \alpha_{02}}{\bar{C}_{11}}\right] \frac{d T}{d r}\right) \times r^{m_{4}} .
\end{gathered}
$$

The Navier equation (15) is nonhomogeneous system of partial differential equations with non-constant coefficients. We assume that $m_{1}=m_{2}$.

\section{Solution of the Navier Equation}

Equation (15) is the Euler differential equation with general and particular solutions.

The general solution is assumed to have the form

$$
u^{g}(r)=B r^{\eta}
$$

Substituting (16) into (15) yields

$$
\left[\eta(\eta-1)+\left(m_{4}+1\right) \eta+\frac{1}{\bar{C}_{11}}\left[\left(m_{4}+1\right) \bar{C}_{12}-\bar{C}_{22}\right]\right]=0
$$

Equation (17) has two roots $\eta_{1}$ to $\eta_{2}$. Thus, the general solutions are

$$
\eta_{1,2}=-\frac{m_{4}}{2} \pm\left(\frac{m_{4}^{2}}{4}-\frac{\left(m_{4}+1\right) \bar{C}_{12}-\bar{C}_{22}}{\bar{C}_{11}}\right)^{1 / 2}
$$

Thus, the general solution is

$$
u^{g}(r)=D_{1} r^{\eta_{1}}+D_{2} r^{\eta_{2}} .
$$

The particular solutions $\mathcal{u}^{P}(r)$ are assumed as

$$
u^{p}(r)=\left(I_{1}+I_{2}\right) r^{\beta+m_{2}} .
$$

Substituting (20) into (18) yields

$$
d_{1} I_{1} r^{\beta+m_{2}-1}+d_{2} I_{2} r^{m_{2}-1}=d_{3} r^{\beta+m_{2}-1}+d_{4} r^{m_{2}-1} .
$$

The complete details for solution of (21) is presented in Appendix B.

The complete solutions for $u(r)$ is sum of the general and particular solutions and are

$$
u(r)=u^{g}(r)+u^{P}(r) .
$$

Thus

$$
u(r)=D_{1} r^{\eta_{1}}+D_{2} r^{\eta_{2}}+\left(I_{1}+I_{2}\right) r^{\beta+m_{2}}
$$

TABle 2

\begin{tabular}{lccccc}
\hline Parameters & $T(a)$ & $T(b)$ & $\sigma_{r r}(a)$ & $\sigma_{r r}(b)$ & $m$ \\
\hline Numerical & $50^{\circ} \mathrm{C}$ & 0 & -50 & 0 & $-1,0,1$ \\
\hline
\end{tabular}

Substituting (23) into (1) and (2), the strains and stresses are obtained as

$$
\begin{aligned}
& \varepsilon_{r r}= \eta_{1} D_{1} r^{\eta_{1}-1}+\eta_{2} D_{2} r^{\eta_{2}-1}+\left(\beta+m_{2}\right)\left(I_{1}+I_{2}\right) r^{\beta+m_{2}-1} \\
& \mathcal{E}_{\theta \theta}=D_{1} r^{\eta_{1}-1}+D_{2} \eta_{2} r^{\eta_{2}-1}+\left(I_{1}+I_{2}\right) r^{\beta+m_{2}} \\
& \sigma_{r r}=\left(\overline { C } _ { 1 1 } \left[\eta_{1} D_{1} r^{\eta_{1}+m_{4}-1}+\eta_{2} D_{2} r^{\eta_{2}+m_{4}-1}\right.\right. \\
&+\left(\beta+m_{2}\right)\left(I_{1}+I_{2}\right) r^{\beta+m_{2}+m_{4}-1} \\
&\left.+\alpha_{01}\left[A_{1}+A_{2}\right]\left(r^{\beta+m_{4}+m_{2}-1}\right)\right] \\
&+\bar{C}_{12}\left[D_{1} r^{\eta_{1}+m_{4}-1}+D_{2} \eta_{2} r^{\eta_{2}+m_{4}-1}+\left(I_{1}+I_{2}\right) r^{\beta+m_{4}+m_{2}}\right. \\
&\left.\left.+2 \alpha_{02}\left[A_{1}+A_{2}\right]\left(r^{\beta+m_{4}+m_{2}-1}\right)\right]\right) e^{i n \theta} \\
& \sigma_{\theta \theta}=\left(\overline { C } _ { 2 2 } \left[D_{1} r^{\eta_{1}+m_{4}-1}+D_{2} \eta_{2} r^{\eta_{2}+m_{4}-1}\right.\right. \\
&\left.+\left(I_{1}+I_{2}\right) r^{\beta+m_{4}+m_{2}}+\alpha_{01}\left[A_{1}+A_{2}\right]\left(r^{\beta+m_{4}+m_{2}}\right)\right] \\
&+\bar{C}_{21}\left[\eta_{1} D_{1} r^{\eta_{1}+m_{4}-1}+\eta_{2} D_{2} r^{\eta_{2}+m_{4}-1}\right. \\
&+\left(\beta+m_{2}\right)\left(I_{1}+I_{2}\right) r^{\beta+m_{2}+m_{4}-1} \\
&\left.\left.+2 \alpha_{02}\left[A_{1}+A_{2}\right]\left(r^{\beta+m_{4}+m_{2}}\right)\right]\right) e^{i n \theta} \cdot
\end{aligned}
$$

To determine the constants $D_{1}$ and $D_{2}$, consider the boundary conditions for stresses given by

$$
\sigma_{r r}\left(a_{1}\right)=-p_{i}, \quad \sigma_{r r}\left(a_{2}\right)=-p_{0} .
$$

\section{Numerical Results and Discussion}

Consider a thick hollow cylinder of inner radius $a=1(\mathrm{~m})$ and outer radius $b=1.2(\mathrm{~cm})$, shown properties are given in Table 1. For simplicity of analysis, we consider that the power law of material properties is the same as $m_{1}=m_{2}=m_{3}=m$. To examine the proposed solution method, two example problems are considered. The example problem may have some physical interpretation.

As the example, consider a thick hollow cylinder where the inside boundary is traction free with given temperature distribution of Table 2. The outside boundary is assumed to be radially fixed with zero temperature. Therefore, the assumed boundary conditions yield of Table 2 .

Figure 1 shows the variations of the temperature along the radial direction for different values of the power law 
index. The figure shows that as the power law index $m$ increases, the temperature decreased.

Figure 2 shows the plot of the radial displacement along the radius. The magnitude of the radial displacement is decreased as the power index $m$ is increased.

The radial and circumferential stresses are plotted along the radial direction and shown in Figures 3 and 4, and the magnitude of the radial stress is increased as $m$ is increased. The hoop stress along the radius decreases for $m, 1$ (similar to thick cylinders made of isotropic materials), due to the acting internal pressure and zero external pressure. For $m<1$, the hoop stress increases as the radius increases, since the modulus of elasticity is an increasing function of the radius. Physically, this means that the outer layers of the cylinder are biased to maintain the stress due to their higher stiffness. There is a limiting value for $m$, where the hoop stress remains almost a constant along the radius. For low values of the ratio $b / a$ (Figures 7 and 8). Figures 5 and 6 show the radial and hoop thermal stresses in the cross-section of the cylinder, respectively, where the pore compressibility coefficient $(B)$ is changed, the other parameters are fixed. Figures 5 and 6 show these stresses based on the pore volume fraction; $(\phi)$ is pore volume per total volume.

Figure 9 shows the radial displacements in the crosssection of the cylinder based on the pore compressibility coefficient $(B)$ changing. Figure 10 also shows these displacements based on the pore volume fraction $(\phi)$ changing.

\section{Conclusions}

In the present work, an attempt has been made to study the problem of general solution for the thermal and mechanical stresses in a thick FGPM hollow cylinder due to the onedimensional axisymmetric steady-state loads. The method of solution is based on the direct method and uses power series, rather than the potential function method. The advantage of this method is its mathematical power to handle both simple and complicated mathematical function for the thermal and mechanical stresses boundary conditions. The potential function method is capable of handling complicated mathematical functions as boundary condition. The proposed method does not have the mathematical limitations to handle the general types of boundary conditions which are usually countered in the potential function method.

\section{Appendices}

\section{A. Compressibility Coefficients and Pore Volume Fraction}

$$
\begin{array}{cc}
e_{1}=\left(S_{12} a^{\beta}-\frac{S_{11}}{m_{3}} A^{\beta+1}\right), & e_{2}=\left(S_{11}\right), \\
e_{3}=\left(S_{22} A^{\beta}-\frac{S_{21}}{m_{3}} A^{\beta+1}\right), & e_{4}=\left(S_{21}\right), \\
\mu=\frac{E_{0}}{2(1+\nu)} . &
\end{array}
$$

$B$ : compressibility coefficient, sometimes called the Skempton pore pressure coefficient.

$$
B=\frac{3\left(\nu_{u}-v\right)}{(1-2 v)\left(1+\nu_{u}\right)}, \quad 0 \leq B \leq 1
$$

$\phi$ : pore volume fraction is pore per unite total volume.

$$
\phi=\frac{\gamma\left(B-k_{f}\right)}{B[(1-\alpha)+k]}
$$

$k_{f}$ and $k$ are bulk modulus of the fluid phase and bulk modulus of the poroelastic medium under the drained condition, respectively.

\section{B. Constants Material}

$$
I_{1}=\frac{d_{4} d_{5}-d_{2} d_{6}}{d_{1} d_{4}-d_{2} d_{3}}, \quad I_{2}=\frac{d_{1} d_{6}-d_{3} d_{5}}{d_{1} d_{4}-d_{2} d_{3}}
$$

where constants $d_{1}$ to $d_{6}$ are given

$$
\begin{aligned}
d_{1}= & \left(m_{2}+1\right)\left(m_{2}\right)+\left(m_{4}+1\right)\left(m_{2}+1\right)+\frac{\left(m_{4}+1\right) \bar{C}_{12}-\bar{C}_{22}}{\bar{C}_{11}} \\
d_{2}= & \left(m_{2}-m_{3}\right)\left(m_{2}-m_{3}-1\right)+\left(m_{4}+1\right)\left(m_{2}-m_{3}\right) \\
& +\frac{\left(m_{4}+1\right) \bar{C}_{12}-\bar{C}_{22}}{\bar{C}_{11}}, \\
d_{3}= & \left\{\left[\left(m_{2}+m_{4}-m_{3}-2\right)-\frac{2 \bar{C}_{12}}{\bar{C}_{11}}\right] \alpha_{01}\right. \\
& \left.+\left[\frac{2(m+m+1) \bar{C}_{12}+\bar{C}_{22}-2\left(m_{3}-1\right) \bar{C}_{12}}{\bar{C}_{11}}\right] \alpha_{02}\right\} A_{n 1}, \\
d_{4}= & \frac{1}{k_{0}}\left\{\left[\left(m_{2}+m_{4}-m_{3}-2\right)-\frac{2 \bar{C}_{12}}{\bar{C}_{11}}\right] \alpha_{01}\right. \\
& \left.+\left[\frac{2\left(m_{1}+m_{4}+1\right) \bar{C}_{12}+\bar{C}_{22}-2\left(m_{3}+1\right) \bar{C}_{12}}{\bar{C}_{11}}\right] \alpha_{02}\right\} A_{n 2}, \\
d_{2}= & C_{22}\left(\eta_{2}+1\right) b^{\eta_{2}+m_{4}-1} \cdot . \\
I_{1}= & \frac{d_{3}}{d_{1}}, \\
d_{1}= & C_{11}\left(\eta_{1}+1\right) a^{\eta_{1}+m_{4}-1}, \\
d_{2}= & C_{22}\left(\eta_{2}+1\right) a^{\eta_{1}+m_{4}-1}, \\
d_{11}\left(\eta_{1}+1\right) b^{\eta_{1}+m_{4}-1}, & d_{4} \\
d_{2} &
\end{aligned}
$$




\section{Nomenclature}

\begin{tabular}{|c|c|}
\hline$a:$ & Inner radius \\
\hline$a_{n}:$ & Thermal constant \\
\hline$b:$ & Outer radius \\
\hline$b_{n}:$ & Thermal constant \\
\hline$S_{i j}:$ & Constant temperature parameters \\
\hline$d_{i}:$ & Mechanical and thermal constants \\
\hline$e_{i}:$ & Mechanical and thermal constants \\
\hline$D_{i j}:$ & Constant mechanical parameters \\
\hline$f_{1}, f_{2}:$ & $\begin{array}{l}\text { Inner and outer temperature boundary } \\
\text { conditions }\end{array}$ \\
\hline$g_{1}, g_{2}, \ldots, g_{8}:$ & $\begin{array}{l}\text { Inner and outer mechanical boundary } \\
\text { conditions }\end{array}$ \\
\hline$k:$ & Thermal conduction coefficient \\
\hline$k_{0}$ : & Material parameter \\
\hline$E:$ & Yong's modulus \\
\hline$E_{0}:$ & Material constant \\
\hline$m_{1}, m_{2}, m_{3}$ & Material parameter \\
\hline$(r, \theta):$ & Cylinder coordinate \\
\hline$T:$ & Cylinder temperature \\
\hline$T_{n}:$ & Coefficient of sine Fourier series \\
\hline$u, v:$ & Displacement components \\
\hline$\alpha:$ & Thermal expansion coefficient \\
\hline$\alpha_{0}:$ & Material constant \\
\hline$\mu:$ & Lame coefficient \\
\hline$v:$ & Poisson's ratio \\
\hline$v_{u}:$ & Undrained Poisson's ratio \\
\hline$p:$ & The pore pressure \\
\hline$M:$ & Biot's modulus \\
\hline$\gamma:$ & Biot's coefficient of effective stress \\
\hline$\delta_{i j}$ & Delta carancker \\
\hline$\zeta:$ & $\begin{array}{l}\text { The variation of fluid content } \\
\text { (undrained } \zeta=0 \text { ) }\end{array}$ \\
\hline$\varepsilon_{i j}:$ & Strain tensor $(i, j)=(r, \theta)$ \\
\hline$\in:$ & Volumetric strain $\left(\in=\varepsilon_{r r}+\varepsilon_{\theta \theta}\right)$ \\
\hline$\sigma_{i j}:$ & Stress tensor $(i, j)=(r, \theta)$ \\
\hline$B:$ & Compressibility coefficient \\
\hline$\phi:$ & $\begin{array}{l}\text { Pore volume fraction is pore per unite } \\
\text { total volume. }\end{array}$ \\
\hline
\end{tabular}

\section{References}

[1] M. A. Biot, "Le problème de la consolidation des matières argileuses sous une charge," Annales de la Societe Scientifique de Bruxelles, vol. B55, pp. 110-113, 1935.

[2] M. A. Biot, "General theory of three-dimensional consolidation," Journal of Applied Physics, vol. 12, no. 2, pp. 155-164, 1941.

[3] R. De Boer, "Highlights in the historical development of the porous media theory: toward a consistent macroscopic theory," Applied Mechanics Reviews, vol. 49, no. 4, pp. 201261, 1996.

[4] E. Detournay and H.-D. A. Cheng, "Fundamentals of poroelasticity," in Comprehensive Rock Engineering: Principles, Practice \& Projects, J. A. Hudson, Ed., pp. 113-171, Pergamon, Oxford, UK, 1993.

[5] R. S. Sandhu and E. L. Wilson, "Finite element analysis of seepage in elastic media," Journal of the Engineering Mechanics Division of the American Society of Civil Engineering, vol. 95, no. 3, pp. 641-652, 1969.
[6] E. Detournay and ,A. H.-D. Cheng, "Fundamentals of poroelasticity," in Comprehensive Rock Engineering: Principles, Practice and Projects, Vol. II, Analysis and Design Method, C. Fairhurst, Ed., chapter 5, pp. 113-171, Pergamon, Oxford, UK, 1993.

[7] Y. Abousleiman and S. Ekbote, "Solutions for the inclined borehole in a porothermoelastic transversely isotropic medium," Journal of Applied Mechanics, vol. 72, no. 1, pp. 102-114, 2005.

[8] P. Y. P. Cheg, "Axismmetric thermal stresses in an anisotropic finite hollow cylinder," Journal of Thermal Stresses, vol. 6, no. 2-4, pp. 197-205, 1983.

[9] B. Bai, "Fluctuation responses of saturated porous media subjected to cyclic thermal loading," Computers and Geotechnics, vol. 33, no. 8, pp. 396-403, 2006.

[10] Y. Wang and E. Papamichos, "An analytical solution for conductive heat flow and the thermally induced fluid flow around a wellbore in a poroelastic medium," Water Resource Reserch, vol. 36, no. 5, pp. 3375-3384, 1994.

[11] Y. Wang and E. Papamichos, "Thermal effects on fluid flow and hydraulic fracturing from wellbores and cavities in lowpermeability formations," International Journal for Numerical and Analytical Methods in Geomechanics, vol. 23, no. 15, pp. 1819-1834, 1999.

[12] A. Ghassemi and Q. Tao, "Influence of coupled chemoporo-thermoelastic processes on pore pressure and stress distributions around a wellbore in swelling shale," Journal of Petroleum Science and Engineering, vol. 67, no. 1-2, pp. 57-64, 2009.

[13] B. Wirth and I. Sobey, "An axisymmetric and fully ${ }_{3} \mathrm{D}$ poroelastic model for the evolution of hydrocephalus," Mathematical Medicine and Biology, vol. 23, no. 4, pp. 363-388, 2006.

[14] D. Yang and Z. Zhang, "Poroelastic wave equation including the Biot/squirt mechanism and the solid/fluid coupling anisotropy," Wave Motion, vol. 35, no. 3, pp. 223-245, 2002.

[15] A. Arora and S. K. Tomar, "Elastic waves along a cylindrical borehole in a poroelastic medium saturated by two immiscible fluids," Journal of Earth System Science, vol. 116, no. 3, pp. 225234, 2007.

[16] Y. Hamiel, V. Lyakhovsky, and A. Agnon, "Coupled evolution of damage and porosity in poroelastic media: theory and applications to deformation of porous rocks," Geophysical Journal International, vol. 156, no. 3, pp. 701-713, 2004.

[17] A. Ghassemi, "Stress and pore prepressure disterbution around apressurized, cooled crack in holw permeability rock " in Proceedings of the 32nd Workshop on Geothermal Reservoir Engineering Stanford University, SGP-TR-183, Stanford University, Stanford, Calif, USA, January 2007.

[18] H. M. Youssef, "Theory of generalized porothermoelasticity," International Journal of Rock Mechanics \& Mining Sciences, vol. 44, no. 2, pp. 222-227, 2007.

[19] S. Jourine, P. P. Valkoo, and A. K. Kronenberg, "Modelling poroelastic hollow cylinderexperiments with realistic boundary conditions," International Journal for Numerical and Analytical Methods in Gwomechanics, vol. 28, no. 12, pp. 11891205, 2004.

[20] M. P. Lutz and R. W. Zimmerman, "Thermal stresses and effective thermal expansion coefficient of a functionally gradient sphere," Journal of Thermal Stresses, vol. 19, no. 1, pp. 39-54, 1996.

[21] R. W. Zimmerman and M. P. Lutz, "Thermal stresses and thermal expansion in a uniformly heated functionally graded cylinder," Journal of Thermal Stresses, vol. 22, no. 2, pp. 177188, 1999. 
[22] M. Jabbari, S. Sohrabpour, and M. R. Eslami, "General solution for mechanical and thermal stresses in a functionally graded hollow cylinder due to nonaxisymmetric steady-state loads," Journal of Applied Mechanics, vol. 79, pp. 493-497, 2002.

[23] R. Poultangari, M. Jabbari, and M. R. Eslami, "Functionally graded hollow spheres under non-axisymmetric thermomechanical loads," International Journal of Pressure Vessels and Piping, vol. 85, no. 5, pp. 295-305, 2008.

[24] M. Shariyat, S. M. H. Lavasani, and M. Khaghani, "Nonlinear transient thermal stress and elastic wave propagation analyses of thick temperature-dependent FGM cylinders, using a second-order point-collocation method," Applied Mathematical Modelling, vol. 34, no. 4, pp. 898-918, 2009.

[25] C. F. Lü, W. Q. Chen, and C. W. Lim, "Elastic mechanical behavior of nano-scaled FGM films incorporating surface energies," Composites Science and Technology, vol. 69, no. 7-8, pp. 1124-1130, 2009.

[26] A. M. Afsar and H. Sekine, "Inverse problems of material distributions for prescribed apparent fracture toughness in FGM coatings around a circular hole in infinite elastic media," Composites Science and Technology, vol. 62, no. 7-8, pp. 10631077, 2002.

[27] D. G. Zhang and Y. H. Zhou, "A theoretical analysis of FGM thin plates based on physical neutral surface," Computational Materials Science, vol. 44, no. 2, pp. 716-720, 2008.

[28] S. A. Fazelzadeh and M. Hosseini, "Aerothermoelastic behavior of supersonic rotating thin-walled beams made of functionally graded materials," Journal of Fluids and Structures, vol. 23, no. 8, pp. 1251-1264, 2007.

[29] Y. Ootao and Y. Tanigawa, "Transient thermoelastic problem of functionally graded thick strip due to non uniform heat supply ," Composite Structures, vol. 63, no. 2, pp. 139-146, 2004.

[30] M. Jabbari, S. Sohrabpour, and M. R. Eslami, "Mechanical and thermal stresses in a functionally graded hollow cylinder due to radially symmetric loads," International Journal of Pressure Vessels and Piping, vol. 79, no. 7, pp. 493-497, 2002.

[31] M. Farid, P. Zahedinejad, and P. Malekzadeh, "Threedimensional temperature dependent free vibration analysis of functionally graded material curved panels resting on twoparameter elastic foundation using a hybrid semi-analytic, differential quadrature method," Materials and Design, vol. 31, no. 1 , pp. 2-13, 2010.

[32] A. Bagri and M. R. Eslami, "Generalized coupled thermoelasticity of functionally graded annular disk considering the Lord-Shulman theory," Composite Structures, vol. 83, no. 2, pp. 168-179, 2008.

[33] B. A. Shariat and M. R. Eslami, "Buckling of thick functionally graded plates under mechanical and thermal loads," Composite Structures, vol. 78, no. 3, pp. 433-439, 2007.

[34] M. Jabbari, A. Bahtui, and M. R. Eslami, "Axisymmetric mechanical and thermal stresses in thick short length FGM cylinder," International Journal of Pressure Vessels and Piping, vol. 2009, no. 5, pp. 296-306, 86.

[35] M. Thieme, K.-P. Wieters, F. Bergner et al., “Titanium powder sintering for preparation of a porous FGM destined as a skeletal replacement implant," Materials Science Forum, vol. 308-311, pp. 374-380, 1999. 

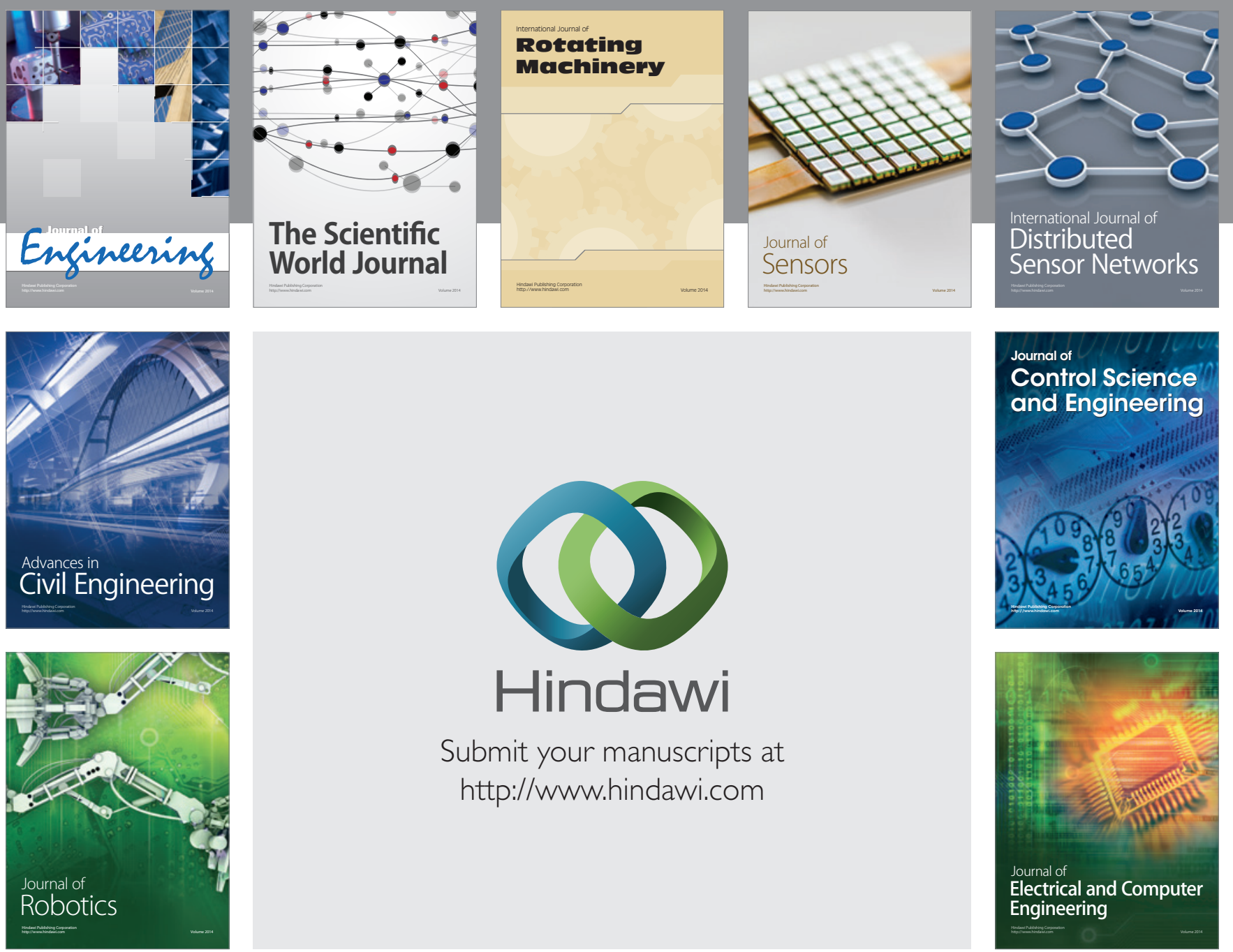

Submit your manuscripts at

http://www.hindawi.com
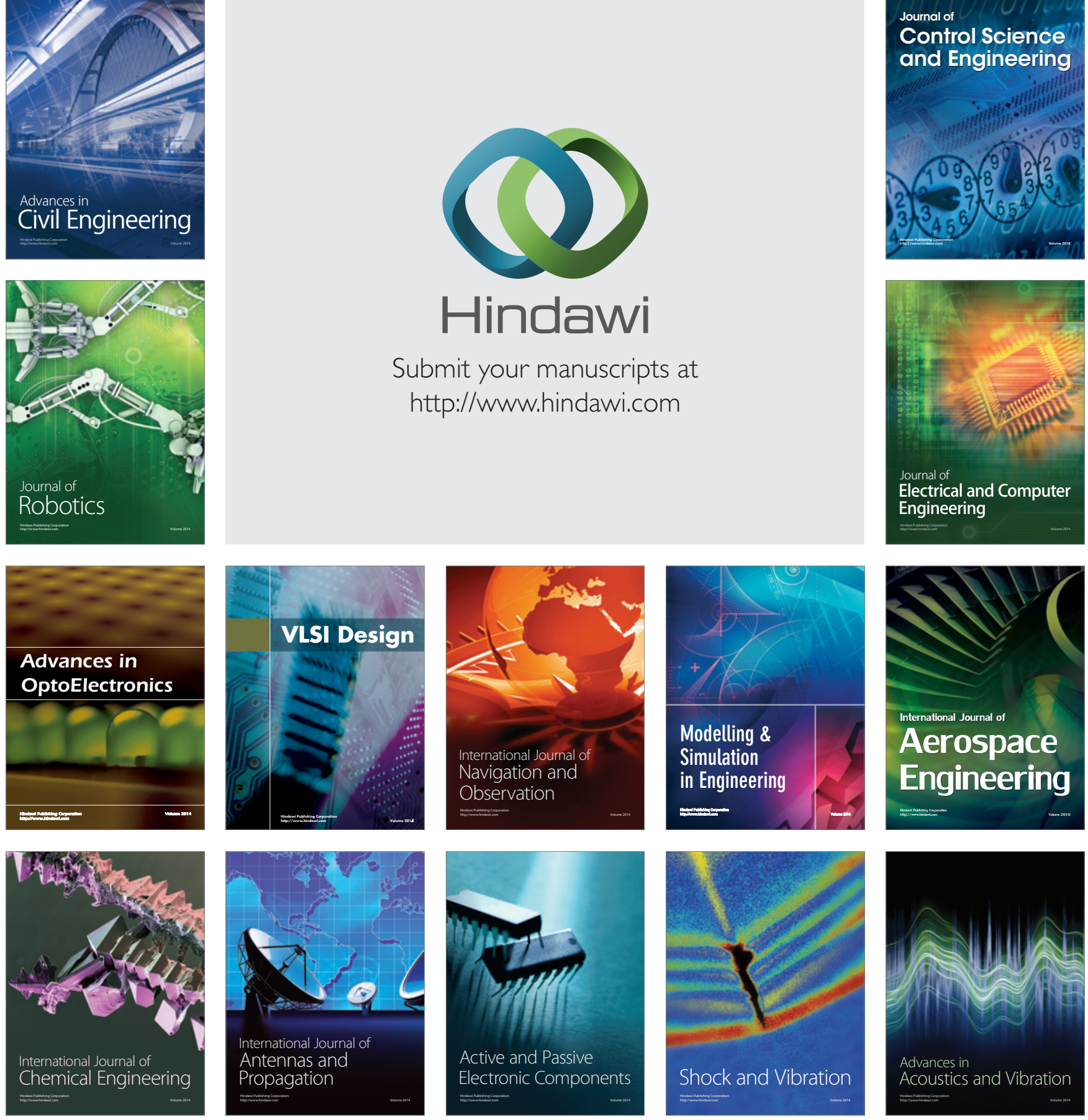